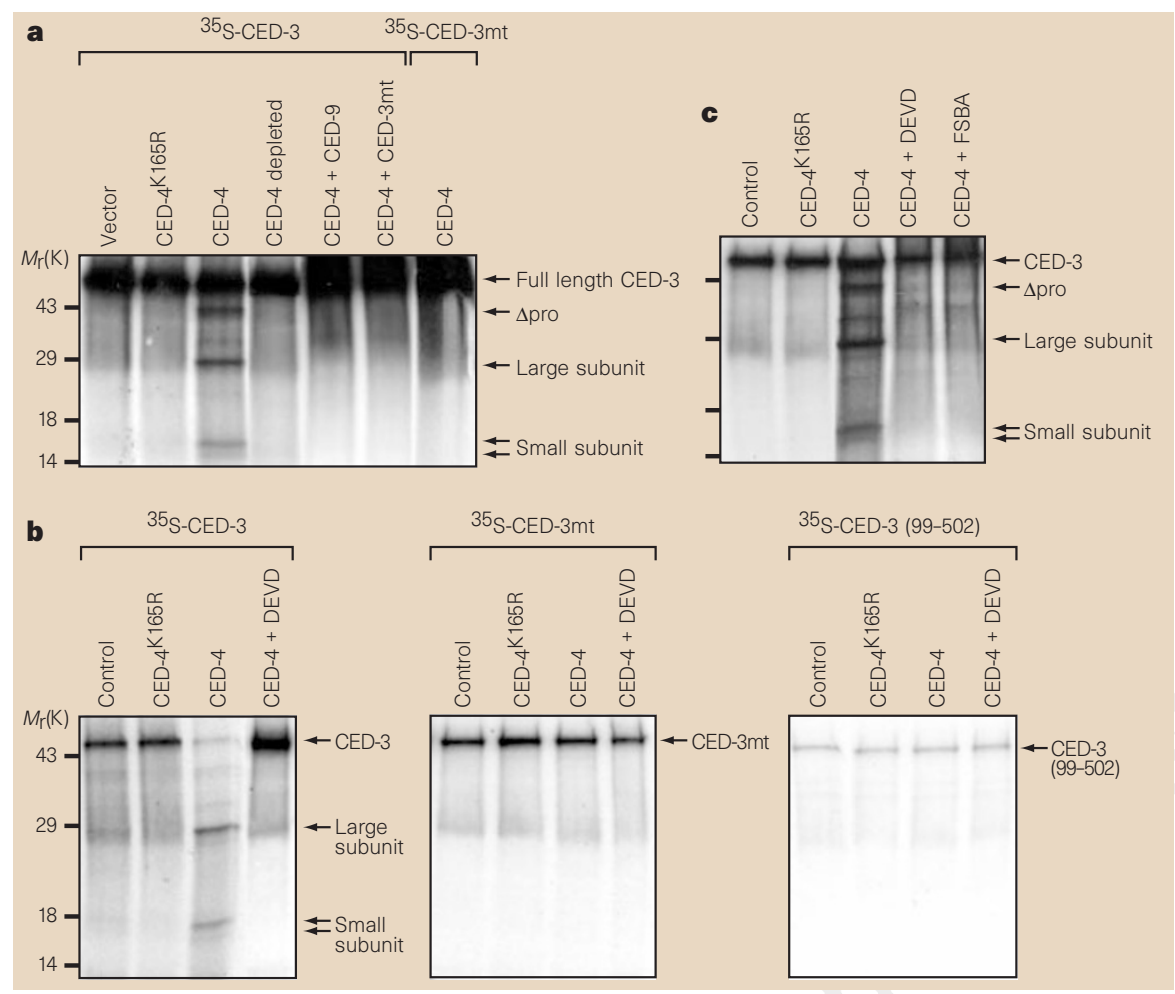

Figure 2 CED-4 facilitates CED-3 auto-activation in vitro. a, Cell extracts expressing CED-4 process in vitro translated, ${ }^{35}$ S-labelled CED-3 but not CED-3mt. Myc/His-CED4 was depleted from an extract derived from CED-4-transfected 293T cells by incubation with $\mathrm{Ni}^{2+}$ beads and further depleted using anti-Myc antibodies and protein G. ${ }^{35}$ S-labelled CED-3 was made by in vitro transcription/translation using the TNT rabbit reticulocyte lysate system (Promega; $30 \mathrm{~min}, 30^{\circ} \mathrm{C}$ ). Prolonged incubation of the in vitro translate triggered auto-processing of CED-3 (ref. 13) and so was avoided. Reactions used $5 \mu \mathrm{l}{ }^{35}$ S-labelled CED-3 and $20 \mu \mathrm{l}$ lysate containing the indicated proteins, incubated for $1 \mathrm{~h}$ at $30{ }^{\circ} \mathrm{C}$. b. Purified CED-4 activates in vitro translated CED-3. CED-4 and CED-4 ${ }^{K 165 R}$ were purified from transfected 293T cells ${ }^{9}$. Reactions used $~ 10-50$ ng purified protein and in vitro translated ${ }^{35}$ S-labelled CED-3, CED-3mt or CED-3(99-502) incubated for $1 \mathrm{~h}$ at $30{ }^{\circ} \mathrm{C}^{10}$. DEVD-aldehyde was at 200 nM. c, CED-4 activation of CED-3 is blocked by FSBA (1 mM). Methods as in $\mathbf{b}$.

of the flax rust-resistance protein L6 (GenBank accession number 862905), a putative P-loop-containing ATPase ${ }^{11}$. The alignment of the C. briggsae CED-4 and L6 sequences consisted of 322 animo-acids with $23 \%$ identical and $43 \%$ similar residues. When the predicted ATPase domain of L6 was used to search the non-redundant database with the PSI-BLAST (position-specific iterative BLAST) program, which enhances BLAST search with profile analysis ${ }^{12}$, the similarity to C. elegans CED-4 was detected at a statistically significant level $\left(P<10^{-5}\right)$. Analysis of a multiple alignment of the CED-4 sequences with those of plant resistance-response gene products homologous to L6 (GenBank accession numbers 862905, 1842251, 1086263, 699495, 1931650 and 1513144) showed a consistent pattern of conservation which was particularly prominent in the P-loop, the putative $\mathrm{Mg}^{2+}$-binding site, and two additional, downstream motifs (unpublished). CED-4 and plantresistance gene products may contain a homologous and probably functionally analogous ATPase domain.

In summary, CED- 4 is central to the regulation of the molecular framework of cell death. It has an intrinsic enzymatic activity which facilitates CED-3 auto-activation and subsequent apoptosis. CED-3 activation by CED-4 requires the activity of the putative CED-4 ATPase domain. The presence of a conserved ATPase domain in CED-4 and the plant resistance-response proteins indicates that there may be similarities between the cell-death machinery and mechanisms in animals and plants.

Arul M. Chinnaiyan, Divya Chaudhary Karen O'Rourke

University of Michigan Medical School,

Department of Pathology, 1301 Catherine Street,

Box 0602, Ann Arbor, Michigan 48109, USA

Eugene V. Koonin

National Center for Biotechnology Information,

National Library of Medicine,

National Institutes of Health, Building 38A,

Bethesda, Maryland 20894, USA

Vishva M. Dixit

Genentech, Inc., 1 DNA Way, M/S-40,

San Francisco, California 94080, USA

e-mail:dixit@gene.com

1. Hengartner, M. O. \& Horvitz, H. R. Curr. Opin. Genet. Dev. 4, 581-586 (1994).

2. Jacobson, M. D. Curr. Biol. 7, R277-R281 (1997).

3. James, C., Gschmeissner, S., Fraser, A. \& Evan, G. I. Curr. Biol. 7, 246-252 (1997).

4. Seshagiri, S. \& Miller, L. Curr. Biol. 7, 455-460 (1997).

5. Hartog, A. F. et al. Biochim. Biophys. Acta 1318, 107-122 (1997).
6. van der Zwet-de Graaff, I., Hartog, A. F. \& Berden, J. A. Biochim. Biophys. Acta 1318, 123-132 (1997).

7. Nagy, A. K. \& Shuster, T. A. J. Neurochem. 65, 1849-1858 (1995). 8. Prescott, M. \& McLennan, A. G. Anal. Biochem. 184, 330-337 (1990).

9. Loo, T. W. \& Clarke, D. M. J. Biol. Chem. 270, 21449-21452 (1995).

10. Chinnaiyan, A. M., O’Rourke, K., Lane, B. R. \& Dixit, V. M. Science 275, 1122-1126 (1997).

11.Lawrence, G. J., Finnegan, E. J., Ayliffe, M. A. \& Ellis, J. G. Plant Cell 7, 1195-1206 (1995).

12. Altschul, S. F. et al. Nucleic Acids Res. (in the press).

13. Hugunin, M., Quintal, L. J., Mankovich, J. A. \& Ghayur, T. J. Biol. Chem. 271, 3517-3522 (1996).

\section{Vaccination onto bare skin}

We report here that applying genetic vectors onto the skin in a relatively non-invasive manner can elicit an immune response against the protein encoded by the vector. This procedure requires no special skill or equipment and so may reduce medical costs and offer a unique method for vaccination.

To elicit a specific immune response, we anaesthetized mice and removed hair and cornified epithelium over a restricted area of abdominal skin using a depilatory (for example Nair $)^{1}$. We pipetted roughly $10^{8}$ plaque-forming units (PFU) of adenovirus vector in a volume of $10-50 \mu$ l onto the preshaved and Nair-treated skin. We allowed the adenovirus vector to incubate with the naked skin until the animal recovered from anaesthesia, which took between $30 \mathrm{~min}$ and $1 \mathrm{~h}$.

After application of AdCMV-hcea, an adenovirus vector encoding the human carcinoembryonic antigen (CEA) gene, onto the skin of mice (strain C57BL/6), we monitored the production of antibodies against the human CEA protein by assaying sera from tail-bleeds. The test sera reacted in western blots with purified human CEA protein, but not with bovine serum albumin (Fig. 1a). Of 24 vaccinated mice, 23 (96\%) produced antibodies against the human CEA protein after a month, indicating that specific antibodies were produced against exogenous proteins encoded by adenovirus vectors as a result of the treatment. Antibodies against adenoviral proteins were also found in some of the treated animals.

To test whether this technique might be generally applicable, we applied AdCMVhgmcsf, an adenovirus vector encoding the human granulocyte macrophage colony stimulating factor (hGM-CSF), in the same way. The test sera reacted with purified human GM-CSF protein (Fig. 1b) in 6 of 14 mice showing that $43 \%$ produced antibodies against the human GM-CSF protein after application of AdCMV-hgmcsf. Pre-immune sera collected before treatment, sera from untreated animals, and sera from animals treated with AdCMV-luc ${ }^{2}$ all failed to react with the human CEA and GM-CSF proteins. 
ลี

CEA BSA Ad

$M_{r}(K)$

$L_{-1}$

$-220$

$-66$

$-46$

123

b

GMCSF BSA Ad $M_{\mathrm{r}}(\mathrm{K})$

$-220$

$-46$

$-14.3$

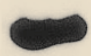

3

Figure $1 \mathrm{a}$, Detection of antibodies to the human CEA protein by western blot analysis. Serum from a vaccinated mouse taken one month after applying AdCMV-hcea to the skin was diluted 1:500 and reacted with purified human CEA protein (provided by T. Strong) and adenoviral proteins separated on a $5 \%$ SDS-polyacrylamide gel. The products were transferred to membranes as in ref. 3. Lane 1, human CEA $(0.5 \mu \mathrm{g})$; lane 2, BSA $(0.5 \mu \mathrm{g})$; lane 3, adenovirus ( $\left.10^{7} \mathrm{PFU}\right)$. b, Detection of antibodies against the human GM-CSF protein. Purified human GM-CSF protein (CalBiochem), separated on a 15\% SDS-polyacrylamide gel, was transferred to membranes and allowed to react with diluted serum. Lane 1, human GM-CSF $(0.25 \mu \mathrm{g})$; lane 2, BSA (0.25 $\mu \mathrm{g})$; lane 3 , adenovirus ( $10^{7} \mathrm{PFU}$ ).

To our knowledge, this is the first demonstration that animals can be vaccinated in a simple, painless, and economical manner by topical application of genetic vectors onto the skin. This strategy may allow the development of vaccines that could be administered by individuals without specialized medical training or equipment.

\section{De-chu Tang*, Zhongkai Shi}

\section{David T. Curiel}

Gene Therapy Program, Department of Medicine

${ }^{*}$ and of Biochemistry and Molecular Genetics,

University of Alabama at Birmingham,

THT 215, 1900 University Boulevard,

Birmingham, Alabama 35294-0006, USA

e-mail:pulm027@uabdpo.dpo.uab.edu

1. Johnston, S. A. \& Tang, D.-c. Meth. Cell Biol. 43, 353-365 (1994).

2. Tang, D.-c., Johnston, S. A. \& Carbone, D. P. Cancer Gene Ther. 1, 15-20 (1994).

3. Tang, D.-c., DeVit, M. \& Johnston, S. A. Nature 356, 152-154 (1992).

\section{Seeing where your hands are}

Some patients with brain damage fail to identify a sensory stimulus presented on the opposite side to their lesion (contralesional) when a competing stimulus is presented on the same side (ipsilesional) ${ }^{1}$. This phenomenon has become known as extinction. It is commonly studied using a single sense such as sight or touch (unimodal extinction) ${ }^{2}$. We have studied a 75-year-old right-handed man (patient GS) who has severe left tactile extinction resulting from damage to the right frontotemporal cortex caused by a stroke. We found that an ipsilesional visual stimulus could induce extinction of a contralesional tactile stimulus (cross-modal extinction). We also found that the visual stimulus operates in a reference system attached to the hand, and not in egocentric coordinates (that is retinal, head or trunkcentred coordinates).

We tested the extinction phenomenon under six experimental conditions. Patient GS sat at a table opposite the experimenter, with his hands positioned on the table surface. In condition 1, tactile stimuli were applied to one or both hands which were placed beneath a cardboard shield to prevent him from viewing them directly. Each stimulus consisted of a light touch to the third finger. In condition 2, the shields were removed and the experimenter applied visual stimuli just in front of GS's third fingers (the same movement as the touch). GS was asked to say how many stimuli he had detected. In both conditions, he reported unilateral stimuli without errors. On stimulation of both sides simultaneously, GS showed marked tactile (1/30 correct trials) but not visual extinction.

In condition 3, GS's left hand was screened with the shield, whereas his right hand was in his view. A tactile stimulus was given to the left hand and a visual stimulus near to the right hand. On single-stimulus trials, his performance was flawless. In contrast, on double-stimulus trials, GS showed a severe left tactile extinction ( $0 / 30$ correct).

Condition 4 was like condition 3, except that GS's right hand was placed behind his back to allow us to assess whether a visual stimulus presented at the same visual field location as in condition 3, but far from the ipsilesional hand, also produces a crossmodal extinction. In both single and double stimulus trials, GS performed correctly.

Condition 5 was like condition 3 except that the visual stimulus was presented far above the right hand, at the level of patient's eye. Again, GS's performance was faultless. Visuotactile extinction therefore manifested itself only when the visual stimulus was pre- sented in an area immediately adjacent to the ipsilesional hand, and disappeared when it was far from the hand.

Condition 6 was like condition 3, except that GS's hands were crossed. He performed normally in single-stimulus trials, but in double-stimulus trials, he showed a severe extinction of the tactile stimuli (1/30 correct) applied to left hand (in right hemispace). Extinction is not modulated by the positions of the hands in space.

Our findings may be explained by referring to the activity of bimodal, visuotactile neurons in the premotor cortex which have receptive fields attached to some relevant body parts ${ }^{3-6}$. Some of these neurons have tactile receptive fields on the hand and corresponding visual receptive fields that extend outward from the tactile field into the space near the hand. As a consequence, activation of these bimodal neurons by a visual stimulus delivered near the hand also activates the corresponding perceptual representation of the hand. Here the simultaneous activation of the somatosensory representation of the left hand (by a tactile stimulus) and of the right hand (by a visual stimulus) produces an extinction of those stimuli presented in the weaker representation, in this case that of the left-hand.

Extinction phenomena (as well as neglect) occur when there is competition between two $^{7,8}$ or more neural representations ${ }^{9}$. In addition, single-neuron studies have shown that visuotactile premotor neurons do not respond when visual stimuli are presented far from the tactile receptive field. This may explain the absence of visuotactile extinction found here and in a previous study $^{10}$, when the visual stimulus was presented far from the ipsilesional hand. Our findings confirm the hypothesis that near space is coded in body-part-centered coordinates, and demonstrate the modular nature of human visual space.

Giuseppe di Pellegrino, Elisabetta Làdavas Department of Psychology, University of Bologna,

Viale Berti Pichat 5, 40127 Bologna, Italy

e-mail:pellegri@psibo.unibo.it

\section{Alessandro Farne'}

Ospedale I.N.R.C.A. 'Fraticini',

Via Massoni 21, 50100 Firenze, Italy

1. Critchley, M. The Parietal Lobes (Edward Arnold, London, 1953)

2. Bisiach, E. \& Vallar, G. in Handbook of Neuropsychology Vol. 7 (eds Boller, F. \& Grafman, J.) 195-222 (Elsevier, Amsterdam, 1988).

3. Gentilucci, M. et al. Exp. Brain Res. 71, 475-490 (1988).

4. Fogassi, L. et al. Exp. Brain Res. 89, 686-689 (1992).

5. Graziano, M. S. A., Yap. G. S. \& Gross, C. G. Science 266, 1054-1057 (1994).

6. Gross, C. G. \& Graziano, M. S. A. in The Cognitive Neurosciences (ed. Gazzaniga, M.) 1021-1034 (MIT Press, Cambridge, MA, 1994).

7. Duncan, J. in Attention and Performance XVI (eds Innui, T. \& McClelland, J. L.) (MIT Press, Cambridge, MA, in the press).

8. di Pellegrino, G., Basso, G. \& Frassinetti, F. Neuropsychologia (in the press).

9. Làdavas, E., Berti, A., Ruozzi, E. \& Barboni, F. Exp. Brain Res. (in the press).

10. Inhoff, A. W., Rafal, R. D. \& Posner, M. J. J. Neurol. Neurosurg. Psychiat. 55, 36-39 (1992). 